\title{
A Scheme of Sustainable Trajectory Optimization for Aircraft Cruise Based on Comprehensive Social Benefit
}

\author{
Lina Ma $\left(\mathbb{D},{ }^{1}\right.$ Yong Tian $\left(\mathbb{D},{ }^{1,2}\right.$ Songtao Yang $\mathbb{D}^{3},{ }^{3}$ Can $\mathrm{Xu} \mathbb{D}^{\mathbb{D}},{ }^{1}$ and Anmin Hao $\mathbb{D}^{1}$ \\ ${ }^{1}$ College of Civil Aviation, Nanjing University of Aeronautics and Astronautics, Nanjing 211100, China \\ ${ }^{2}$ State Key Laboratory of Air Traffic Management System and Technology, Nanjing 210007, China \\ ${ }^{3}$ School of Mechanical Engineering, University of Leeds, Leeds LS2 9JT, UK \\ Correspondence should be addressed to Yong Tian; tianyong@nuaa.edu.cn
}

Received 25 May 2021; Accepted 21 June 2021; Published 2 July 2021

Academic Editor: Maria Alessandra Ragusa

Copyright (c) 2021 Lina Ma et al. This is an open access article distributed under the Creative Commons Attribution License, which permits unrestricted use, distribution, and reproduction in any medium, provided the original work is properly cited.

\begin{abstract}
The increasing demand for environmentally friendly and passenger-favored flight operation requires a systematic scheme of sustainable trajectory optimization for the aircraft cruise. This paper achieves it by proposing an innovative performance framework based on the comprehensive benefit to the society considering both economic and noneconomic ones, following which the sustainable trajectory optimization problem is modeled by discretization. A method combining forward recurrence and memoization operation, called memoization dynamic programming, is developed to solve the model with computational efficiency. Working with real-world operational data of a typical flight route, we demonstrate the effectiveness of the proposed scheme at different levels and explore the difference in its performance due to meteorological conditions, aircraft type, and time horizon. The scheme is proved to perform robustly in comprehensive performance with a stable benefit rate of about $8 \%$ through sensitivity analysis, by which we find that it is relatively better for the flights cruising on business route with a load factor of $85 \%$. Tradeoff results suggest that the systematic consideration of both the economic and noneconomic performance contributes to improved integrated sustainability. In particular, the optimal comprehensive performance at a monthly level can be obtained when accepting an additional \$26,500 economic cost.
\end{abstract}

\section{Introduction}

The aircraft trajectory optimization (ATO) is one of the problems aiming to find the optimal state and control sequences so as to optimize the predefined objective index [1]. Commercial aircraft operators have been most committed to ATO to gain reductions in fuel consumption and time cost for economic savings [2] over the past several decades. With the growing demand for aviation service according to the International Civil Aviation Organization (ICAO), the increasing requirement for sustainable transport leads to the necessity of taking some noneconomic factors other than economic ones into consideration during ATO.

Rather than natural events, the pollution originated by human activities is the most accused in global warming [3]. Particularly, the increasing air transport has led to gradually prominent environmental issues like air pollution in the last decade. It is reported that $24 \%$ of the global nitrogen oxide $\left(\mathrm{NO}_{x}\right)$ emissions and $13 \%$ of the transported carbon dioxide $\left(\mathrm{CO}_{2}\right)$ emissions can be attributed to aviation [4], and the coverage of contrails is also expected to rise to $0.22 \%$ by the middle of this century [5]. In view of environmental concerns, some scholars have worked to obtain environmentally friendly trajectories through ATO. As developed by Hartjes et al. [6], it can be achieved by minimizing the flight time in the area where contrails may take place. With the consideration of both $\mathrm{CO}_{2}$ emissions and contrail formation, Tian et al. [7] defined the green direct operating cost (GDOC) and succeeded in obtaining the optimal cruise altitude and speed with the minimum GDOC. The four-dimension (4D) green trajectory optimization was further implemented by their group [8] taking more factors such as $\mathrm{NO}_{x}$ emissions and other harmful gases into account. Furthermore, other considerations about operation safety and social impact have 
also been introduced into ATO by achieving collision avoidance [9] and noise abatement [10].

From the perspective of market development, attention should also be paid to passengers in aircraft trajectory planning. Improving passengers' benefit will help airlines to enhance passengers' satisfaction and build their loyalty in the increasingly fierce competition, especially under some external shocks such as COVID-19. Kos Koklic et al. [11] found that $35 \%$ of passengers make choices based on their past flight experience, satisfaction evaluation, and the possibility of delay. It means that the passenger-favored strategy of aircraft operation is conducive to boosting marketing gains. However, so far to the best of the author's knowledge, there is no work that has been reported to consider passengers' benefit (or loss) in the process of trajectory decision-making.

As for the methods that formulate and solve the ATO problems, the widely used mathematical programming approaches such as the direct collocation [12], the nonlinear integer programming (NLIP) [13], the sequential programming [14], and the geometric programming [15] can be found feasible in the literature. However, they are not competent enough for practical application due to time and space complexities. Recently, a series of alternatives to the mathematical programming methods have emerged to generate near-optimal solutions of ATO based on metaheuristics techniques, including the genetic algorithm (GA) [16], the simulated annealing (SA) [17], the particle swarm optimization (PSO) [18], and the differential evolution algorithm (DEA) [19]. Although these metaheuristics methods have an advantage in reducing time consumption based on heuristic rules, the optimality of obtained solutions is questionable for the randomness in choosing the initial population [20].

Toward the goal of obtaining a preferable aircraft trajectory strategy that improves the integrated sustainability and benefits to the overall society in acceptable computational time, we develop an effective and robust scheme of sustainable aircraft trajectory optimization (SATO) for the cruise phase, in which the spent time accounts for the majority of the total flight and the greenhouse effect of aircraft exhausts is significantly amplified [21]. The contribution of our work can be summarized in the following three aspects.

\subsection{Model}

(i) A novel performance framework of SATO is established with a view to benefiting all of the airlines, passengers, and the atmosphere environment, and the travel time value for passengers is involved in ATO work for the first time

(ii) The problem of SATO is transformed into a discrete dynamic system for modeling, following which it is formulated as a process of multistage optimization decision

\subsection{Method}

(i) A practical and tractable dynamic programming(DP-) based method with forward recurrence is adopted to find the globally optimal solutions of the SATO model

(ii) Two memos are introduced when processing, forming a memoization dynamic programming (MDP) algorithm, to obtain computational complexity reduction and solution efficiency improvement by avoiding massive double operations

\subsection{Application}

(i) Example experiments are carried out for the flights cruising on the busiest domestic flight route in China; numerical results at different levels are given by which various operating durations and aircraft types are compared in terms of the performance of SATO

(ii) The robustness of the proposed SATO scheme is discussed by sensitivity analysis, and the tradeoff between the economic and noneconomic benefit is also explored for decision support in the application

The remainder of this paper is constructed as follows. Section 2 establishes a SATO performance framework which innovatively takes the comprehensive social benefit into account. In Section 3, the proposed SATO problem is modeled and formulated by discretization, following which an MDP method for efficiently obtaining the optimal solution is presented in detail. Section 4 shows and discusses the results of a case study using real-world operational data. Finally, Section 5summarizes the research with concluding remarks and gives an outlook to the future work.

\section{Performance Framework of SATO for Aircraft Cruise}

In this section, we establish a performance framework of SATO for aircraft cruises to measure the comprehensive benefit that trajectory optimization brings to the overall society. The framework includes three benefit indices considering air carriers, the atmosphere environment, and passengers, respectively.

2.1. Economic Benefit Index. The first benefit index is built to measure the optimization efficiency in reducing airlines' DOC that enhances the economic sustainability of aircraft operation.

2.1.1. Fuel Cost. Fuel consumption generates an important part of airlines' DOC and is also the main source of aviation environmental pollutants. According to the Base of Aircraft Data (BADA) released by EUROCONTROL [22], the 
aircraft fuel flow rate during the cruise, FFRin $\mathrm{kg} / \mathrm{min}$, can

be modeled as

$$
\mathrm{FFR}=\frac{C_{f_{1}} \cdot C_{f c r}\left(\left(1+V_{\mathrm{TAS}}\right) / C_{f_{2}}\right)}{1000} \cdot\left[\frac{\rho \cdot\left(1.944 V_{\mathrm{TAS}}\right)^{2} \cdot S \cdot C_{D_{0}, \mathrm{CR}}}{2}+\frac{2 m^{2} \cdot g_{0}^{2} \cdot C_{D_{2}, \mathrm{CR}}}{\rho \cdot\left(1.944 V_{\mathrm{TAS}}\right)^{2} \cdot S}\right],
$$

where $V_{\text {TAS }}$ is the true airspeed (TAS) in knot (kt), $C_{f c r}$ indicates the correction coefficient of cruise fuel flow, $C_{f_{1}}$ and $C_{f_{2}}$ are the first and the second thrust specific fuel consumption coefficients, respectively, $C_{D_{0}, \mathrm{CR}}$ and $C_{D_{2}, \mathrm{CR}}$ represent the parasitic and the induced drag coefficient during cruise respectively, $\rho$ denotes the air density in $\mathrm{kg} / \mathrm{m}^{3}$, Sis the area of aircraft wing surface in $\mathrm{m}^{2}, m$ denotes the aircraft reference mass in $\mathrm{kg}$, and $g_{0}$ is the local acceleration of gravity in $\mathrm{m} / \mathrm{s}^{2}$.

As formulated by equation (2), the total fuel cost Cost $_{\text {fuel }}$ is essentially the product of the fuel cost coefficient $C_{F}$ and the total fuel consumption, which can be obtained by calculating the integral of the fuel flow rate at each moment:

$$
\text { Cost }_{\text {fuel }}=C_{F} \int_{0}^{T_{c}} \operatorname{FFR}(t) \mathrm{d} t,
$$

where $T_{c}$ is the total time of aircraft cruise and FFR $(t)$ is the fuel flow rate at the moment of $t$.

2.1.2. Time-Related Cost. The time-related cost, Cost $_{\text {time }}$, constitutes the other part of the airline's DOC besides fuel cost. Its value depends on the operation time and the time cost coefficient $C_{T}$ as described in equation (3) in which $C_{T}$ is related to $C_{F}$ and another parameter as equation (4) formulates

$$
\begin{aligned}
\text { Cost }_{\text {time }} & =C_{T} \cdot T_{c}, \\
C_{T} & =\mathrm{CI} \cdot C_{F},
\end{aligned}
$$

where CI is the mentioned parameter, representing the cost index that aircraft carriers adopt to control their DOC for the specific flight phase and aircraft type. Generally, a smaller CI means relatively less fuel burn while more time consumption.

In terms of the efficiency of our optimization work, the economic performance of SATO is reflected by the resulting changes in airlines' DOC. Note that the expected benefit is always derived from the negative variation of cost; the economic benefit index, EBI, can be defined as

$$
\mathrm{EBI}=\Delta \text { Cost }_{\text {fuel }}+\Delta \text { Cost }_{\text {time }}
$$

where $\Delta$ Cost $_{\text {fuel }}$ and $\Delta$ Cost $_{\text {time }}$ are the reduction of fuel cost and time-related cost, respectively, after optimization.

2.2. Green Benefit Index. In the SATO performance framework, the contribution to environmental sustainability is quantified by a green benefit index that considers the greenhouse effect generated by major aviation pollutants including $\mathrm{CO}_{2}$ and $\mathrm{NO}_{x}$ emissions and contrails.
2.2.1. Aviation Pollutants. It has been mentioned previously that aircraft operations are largely to blame for the increasingly severe issue of global warming. The major aviation pollutants which can be quantified as below can be effectively reduced, if not completely eliminated, by optimizing the trajectory strategy.

(1) $\mathrm{CO}_{2}$ Emissions. As equation (6) indicates, the value of $\mathrm{CO}_{2}$ emissions, $E_{\mathrm{CO}_{2}}$, depends on the fuel consumption and the $\mathrm{CO}_{2}$ emission index $\mathrm{EI}_{\mathrm{CO}_{2}}$ that values constantly given a specific aircraft type.

$$
E_{\mathrm{CO}_{2}}=\mathrm{EI}_{\mathrm{CO}_{2}} \int_{0}^{T_{c}} \operatorname{FFR}(t) \mathrm{d} t
$$

(2) $\mathrm{NO}_{x}$ Emissions. The value of $\mathrm{NO}_{x}$ emissions is also related to the fuel consumption and the gas emission index. However, the value of the $\mathrm{NO}_{x}$ emission index is affected by the real-time atmospheric conditions and fuel flow rate in addition to the aircraft type, meaning that it will change constantly during operation. Therefore, the total $\mathrm{NO}_{x}$ emissions, $E_{\mathrm{NO}_{x}}$, should be the integral of emissions at all times as equation (7) shows

$$
E_{\mathrm{NO}_{x}}=\int_{0}^{T_{c}} \mathrm{EI}_{\mathrm{CO}_{2}} \cdot \operatorname{FFR}(t) \mathrm{d} t
$$

where $\mathrm{EI}_{\mathrm{NO}_{x}}(t)$ is the $\mathrm{NO}_{x}$ emission index at the moment of $t$; it can be obtained through interpolation and modification based on atmospheric conditions [23].

(3) Contrail Formation. Contrails form due to exhaust emissions during aircraft cruises and cause a more serious greenhouse effect. They will appear when the requisites that (i) $\mathrm{RH}_{\text {critical }} \leq \mathrm{RH}_{w} \leq 100 \%$ and (ii) $\mathrm{RH}_{w i} \geq$ $100 \%$ are satisfied, where $\mathrm{RH}_{\text {critical }}$ is the critical relative humidity, $\mathrm{RH}_{w}$ is the relative humidity to water, and $\mathrm{RH}_{i}$ donates that to ice, and it can be computed from $\mathrm{RH}_{w}$ and the atmospheric temperature, $T$, as equation (8) describes [24].

$$
\mathrm{RH}_{i}=\mathrm{RH}_{w} \frac{6.0612 \exp [18.102 T /(2.49 .52+T)]}{6.1162 \exp [22.577 T /(237.78+T)]}
$$

Once atmospheric conditions meet the two requisites, the length of the generated contrail, $L_{\text {Con }}$, in the nautical mile (nm) can be written as

$$
L_{\text {Con }}=V_{\mathrm{TAS}} \cdot \frac{T_{c}}{60} .
$$


2.2.2. Equivalent Cost of Temperature Change. The absolute global temperature potential (AGTP) is employed in this paper to uniformly measure the environmental impact of different pollutants from the perspective of temperature change. The total caused temperature change effect on the atmosphere environment with an $H$-year time horizon, $\mathrm{TC}(H)$, can be comprehensively measured by a linear function:

$$
\begin{aligned}
\operatorname{TC}(H)= & \operatorname{AGTP}_{\mathrm{CO}_{2}}(H) \cdot E_{\mathrm{CO}_{2}}+\operatorname{AGTP}_{\mathrm{NO}_{x}}(H) \cdot E_{\mathrm{NO}_{x}} \\
& +\operatorname{AGTP}_{\mathrm{Con}}(H) \cdot L_{\mathrm{Con}},
\end{aligned}
$$

where $\operatorname{AGTP}_{\mathrm{CO}_{2}}(H), \operatorname{AGTP}_{\mathrm{NO}_{x}}(H)$, and $\operatorname{AGTP}_{\text {Con }}(H)$ are the AGTP values of $\mathrm{CO}_{2}, \mathrm{NO}_{x}$, and contrail, respectively [25].

In order to mitigate the issue of global warming, the international community has widely adopted the method of internalizing external effects by charging for $\mathrm{CO}_{2}$ emissions according to the social cost of carbon (SCC). Chen et al. [26] further parameterize the equivalent cost of temperature change per Kelvin (ECK) through SCC to evaluate the environmental impacts of aircraft operation. The value of ECK in an $H$-year time horizon, $\operatorname{ECK}(H)$, is formulated as

$$
\operatorname{ECK}(H)=\frac{1}{1000} \frac{\operatorname{SCC}}{\operatorname{AGTP}_{\mathrm{CO}_{2}}(H)} .
$$

In this paper, we employ it to monetize the environmental benefit of SATO. The total equivalent cost of temperature change caused by aviation pollutants in the time horizon of $H$ years, Cost ${ }_{\text {env }}(H)$, is calculated by

$$
\text { Cost }_{\text {env }}(H)=\operatorname{ECK}(H) \cdot \mathrm{TC}(H) \text {. }
$$

Similar to EBI, we establish a green benefit index, GBI, as

$$
\mathrm{GBI}=\Delta \operatorname{Cost}_{\mathrm{env}}(H)=\mathrm{ECK}(H) \cdot \Delta \mathrm{TC}(H),
$$

where $\triangle \mathrm{TC}(H)$ refers to the reduction of the total temperature change with a time horizon of $H$ years and $\Delta \operatorname{Cost}_{\text {env }}(H)$ is the corresponding decrement in equivalent cost of temperature change due to $\Delta \mathrm{TC}(H)$.

2.3. Passenger Benefit Index. As mentioned previously, the benefit that passengers obtain from SATO is critical to the marketing sustainability of the industry development, for which a passenger benefit index is introduced into the performance framework in this paper through the travel time value.

2.3.1. Travel Time Value. Time consumption not only affects the time-related cost in airline's DOC but is also the biggest expenditure for passengers in addition to the money spent on paying the fare. In addition to bringing direct economic losses [27], flight delays have been well believed to seriously reduce passenger satisfaction, making it convincing that the aircraft trajectory strategy will exert influence on passengers' benefit by changing the operating time. But how to quantify the impact of change in operating time on passengers' benefit? The value of travel time (VTT) is introduced to achieve it in this paper. As defined by Shao and Chen [28], VTT indicates the monetary performance of the benefit increase due to the march of time and the benefit loss due to the unproductive consumption of it.

As for the specific calculation of VTT, it is mostly performed based on production accounting, income accounting, or the logit model of travel willingness. The production accounting method is from the perspective of macroeconomics and holds an opinion that travel time serves as one of the production factors, which will improve the gross domestic product (GDP) when invested into production activities. Focusing on the opportunity cost of passengers, the reduction of personal income for their spending time on traveling is regarded as VTT in the method of income accounting. Also, many scholars have considered people's subjective intention and explored their preferences through sample surveys, based on which a series of logistic regression models of travel utility have been established to calculate VTT combined with statistical analysis [29, 30].

2.3.2. Passenger Loss for Time Consumption. In this study, we employ VTT to measure the loss of passengers due to time consumption, based on which a passenger benefit index is constructed aiming to make up for the lack of attention to passengers in the existing ATO work. It is universally acknowledged that aviation passengers are divided into two categories including business passengers and leisure ones according to their traveling purposes [31]. Generally, business passengers are relatively more sensitive to time compared with the other, indicating a difference in passenger's loss caused by the same amount of time consumption. For this reason, we adopt various methods to calculate VTT according to the passenger type.

For the passengers traveling on business, the time they spend on the itinerary is essentially working time, which can be considered as a production input that contributes to the total output. Therefore, the method of production accounting as equation (14) describes is used to calculate VTT for them:

$$
v_{b}=\frac{G}{W \cdot T_{w}}
$$

where $v_{b}$ is the value of unit travel time for business passengers in $\$ / h, G$ represents the value of the annual gross domestic product (GDP), and $W$ and $T_{w}$ are the population of the labor force and the personal effective working hours in the corresponding year, respectively.

For leisure passengers, spending time in the cabin means giving up a certain amount of economic income from work. According to the well-known time allocation theory firstly proposed by Becker [32], the reason why individuals allocate time to nonwork activities is that the value of leisure time is equivalent to the wage rate at work. Hence, the result of VTT for leisure passengers can be obtained through the income accounting method according to 


$$
v_{l}=\frac{I_{a}}{T_{w}},
$$

where $v_{l}$ is the value of unit travel time for leisure passengers and $I_{a}$ represents per capita annual income.

Given the results about VTT, the total loss for time consumption of different kinds of passengers, $\operatorname{Loss}_{T_{c}}$, can be monetized as

$$
\operatorname{Loss}_{T_{c}}=\left[p_{b} \cdot v_{b}+\left(1-p_{b}\right) \cdot v_{l}\right] \cdot N_{p} \cdot \frac{T_{c}}{60},
$$

where $N_{p}$ denotes the passenger number on the flight and $p_{b}$ is the proportion of business passengers in $N_{p}$.

In order to quantify passengers' benefit from SATO, we formulate a passenger benefit index, PBI, as equation (17) based on their loss reduction through time saving:

$$
\mathrm{PBI}=\Delta \operatorname{Loss}_{T_{c}}=\left[p_{b} \cdot v_{b}+\left(1-p_{b}\right) \cdot v_{l}\right] \cdot N_{p} \cdot \frac{\Delta T_{c}}{60},
$$

where $\Delta \operatorname{Loss}_{T_{c}}$ is the total reduction in passengers' loss for time consumption.

To sum up, the total social cost of aircraft operation is composed of the airlines' DOC including fuel cost and time cost, the equivalent cost of temperature change due to the greenhouse effect on the atmospheric environment, and the total passenger loss for time consumption. In order to achieve an improvement of the comprehensive social benefit, there are three benefit indices including EBI, GBI, and PBI in the performance framework of SATO, and they collaboratively constitute a comprehensive benefit index CBI as equation (18) describes.

$$
\mathrm{CBI}=\mathrm{EBI}+\mathrm{GBI}+\mathrm{PBI} .
$$

\section{Implementation Scheme of SATO for Aircraft Cruise}

Based on the performance framework, this section presents the systematic implementation scheme of SATO for aircraft cruises, including mathematically formulating the problem and developing a solution methodology in the premise of assumptions.

3.1. Assumptions. As a constrained optimal control problem, the process of SATO for aircraft cruise can be achieved by finite-dimensional parametrization to transform the continuous system into a discrete dynamic one based on segments. In order to facilitate the problem formulation, the following assumptions are adopted in this paper: (i) the aircraft is considered as a particle, (ii) the decisions about speed and flight level for each segment are implemented and completed at the segment beginning, and (iii) the speed remains unchanged when the aircraft transforms flight level, ensuring the negligibility of operating time variation.

3.2. Mathematic Formulation. The essence of the studied problem is to achieve the predefined objective by adjusting the trajectory strategy in terms of speed and flight level on each cruise route. The problem can be formulated as a process of multistage optimization decision, and the decision variables, objective function, and constraints will be presented with the detailed notations of the formulation defined as follows:

\section{Notation}

$J$ : set of segments on the cruise route $j$ : index of segments on the cruise route, $j \in J$

$N_{j}$ : number of segments on the cruise route

$N_{p}$ : number of passengers on the flight

$l_{j}$ : length of $j$, in $\mathrm{nm}$

$v_{j}^{\text {ori }}$ : original cruise speed on $j$, in kt

$v_{j}$ : optimized cruise speed on $j$, in kt

$h_{j}^{\text {ori }}$ : original cruise flight level on $j$, in $\mathrm{ft}$

$h_{j}$ : optimized cruise flight level on $j$, in $\mathrm{ft}$

$\mathrm{FC}_{j}^{\text {ori }}$ : fuel consumption on $j$ with the original trajectory, in $\mathrm{kg}$

$\mathrm{FC}_{j}$ : fuel consumption in $\mathrm{kg}$ on $j$ with the optimized trajectory, in $\mathrm{kg}$

$\mathrm{EI}_{\mathrm{NO}_{x}, j}^{\text {ori }}$ : emission index of $\mathrm{NO}_{x}$ on $j$ with the original trajectory, in $\mathrm{kg} / \mathrm{kg}$

$\mathrm{EI}_{\mathrm{NO}_{x}, j}$ : emission index of $\mathrm{NO}_{x}$ on $j$ with the optimized trajectory, in $\mathrm{kg} / \mathrm{kg}$

$\beta\left(h_{j}^{\text {ori }}\right)$ : contrail formation coefficient on $h_{j}^{\text {ori }}$, it values 1 if contrails exist on $h_{j}^{\text {ori }}$ and values 0 otherwise

$\beta\left(h_{j}\right)$ : contrail formation coefficient on $h_{j}$, it values 1 if contrails exist on $h_{j}$ and values 0 otherwise

$V_{j}$ : lower bound of speed for aircraft cruise on $j$

$\overline{\overline{V_{j}}}$ : upper bound of speed for aircraft cruise on $j$

$\mathrm{FL}_{j}$ : lower bound of flight level for aircraft cruise on $j$

$\overline{\overline{\mathrm{FL}_{j}}}$ : upper bound of flight level for aircraft cruise on $j$

$m_{v}$ : maximum absolute variation on speed for aircraft cruise

$\mu_{v}$ : step size of absolute variation on speed for aircraft cruise

$m_{h}$ : maximum absolute variation on flight level for aircraft cruise

$\mu_{h}$ : step size of absolute variation on flight level for aircraft cruise 
3.2.1. Decision Variables. Quantities (19) and (20) declare the decision variables and the associated domains of the problem.

$\gamma_{j}= \begin{cases}1, & \text { adjust the speed at the beginning of segment } j, \\ 0, & \text { otherwise, }\end{cases}$

$\lambda_{j}= \begin{cases}1, & \text { adjust the flight level at the beginning of segment } j, \\ 0, & \text { otherwise, }\end{cases}$
3.2.2. Objective Function. In light of the performance framework established in Section 2, the objective function used in optimization aims to maximize the comprehensive benefit to the overall society as

$$
\operatorname{Max} \mathrm{CBI}=\operatorname{Max}(\mathrm{EBI}+\mathrm{GBI}+\mathrm{PBI}) .
$$

The summed three items correspond to the indices in the performance framework working to measure the effect of rescheduling the origin trajectory, which in turn are treated as the baseline for computing the obtained benefit (cost reduction) resulting from SATO. Accordingly, the specific consumption for them is denoted in

$$
\begin{aligned}
\mathrm{EBI}= & \sum_{j \in J}\left[C_{F} \cdot\left(\mathrm{FC}_{j}^{\text {ori }}-\mathrm{FC}_{j}\right)+60 C_{T} \cdot \frac{l_{j}}{v_{j}-v_{j}^{\text {ori }}}\right] \\
\mathrm{GBI}= & \frac{\mathrm{SCC}}{1000 \cdot \mathrm{AGTP}_{\mathrm{CO}_{2}}(H)} \cdot\left[\mathrm{AGTP}_{\mathrm{CO}_{2}}(H) \cdot \mathrm{EI}_{\mathrm{CO}_{2}} \sum_{j \in J}\left(\mathrm{FC}_{j}^{\text {ori }}-\mathrm{FC}_{j}\right)+\mathrm{AGTP}_{\mathrm{NO}_{x}}(H) \sum_{j \in J}\left(\mathrm{EI}_{\mathrm{NO}_{x}, j}^{\text {ori }} \cdot \mathrm{FC}_{j}^{\text {ori }}-\mathrm{EI}_{\mathrm{NO}_{x}, j} \cdot \mathrm{FC}_{j}\right)\right. \\
& \left.+\mathrm{AGTP}_{\mathrm{Con}}(H) \sum_{j \in J}\left(\beta\left(h_{j}^{\text {ori }}\right)-\beta\left(h_{j}\right)\right) \cdot l_{j}\right],
\end{aligned}
$$$$
\mathrm{PBI}=\sum_{j \in J} N_{p} \cdot l_{j} \cdot \frac{\left[\left(\left(p_{b} \cdot G\right) /\left(W \cdot T_{w}\right)\right)+\left(1-p_{b}\right) \cdot\left(I_{a} / T_{w}\right)\right]}{v_{j}-v_{j}^{\text {ori }}} .
$$

3.2.3. Constraints. The considered constraints involve three aspects about airspace operation, aircraft maneuverability, and control workload.

(1) Airspace operation:

$$
\begin{gathered}
\underline{V_{j}} \leq v_{j} \leq \overline{V_{j}}, \quad \forall j \in J, \\
\underline{\mathrm{FL}_{j}} \leq h_{j} \leq \overline{\mathrm{FL}_{j}}, \quad \forall j \in J .
\end{gathered}
$$

Equations (25) and (26) describe the airspace limits specified in operation. They stipulate the bounds of cruise speed and flight level for each segment.

(2) Aircraft maneuverability:

$$
\begin{aligned}
& \left|v_{j+1}-v_{j}\right| \leq m_{v}, \quad \forall j, j+1 \in J, \\
& \frac{\left|v_{j+1}-v_{j}\right|}{\mu_{v}} \in N, \quad \forall j, j+1 \in J, \\
& \left|h_{j+1}-h_{j}\right| \leq m_{h}, \quad \forall j, j+1 \in J, \\
& \frac{\left|h_{j+1}-h_{j}\right|}{\mu_{h}} \in N, \quad \forall j, j+1 \in J .
\end{aligned}
$$

Taking the aircraft maneuverability into account due to safety concerns, equations (27) and (28) enforce that the absolute variation on speed is not larger than $m_{v}$ if taking the decision to adjust speed $\left(\gamma_{j}=1\right)$ and can only value following a specified step size of $\mu_{v}$. Equations (29) and (30) guarantee similar restrictions when transforming flight levels $\left(\lambda_{j}=1\right)$.

(3) Control workload:

$$
\gamma_{j}+\lambda_{j} \neq 2, \quad \forall j \in J
$$

In order to decrease the workload of air traffic controllers, equation (31) ensures the actions of adjusting speed and altitude cannot be performed at the same time for each segment.

3.3. Solution Methodology. The SATO problem formulated above has a structure with Markov property and can be solved using the DP method like other Markov decision processes. Developed firstly by Richard Bellman in 1952, DP solves a complex optimization problem by recursively splitting it into simpler subproblems with similar features until the subproblems are resoluble, and the global solution is obtained by combining results of subproblems [33]. However, the classic DP is usually impracticable for the 
curse of dimensionality when applied to specific programs [34]. In this regard, an improved DP called memoization dynamic programming (MDP) is developed to efficiently achieve SATO, and it will be developed in three steps: (i) problem discretization, (ii) forward recurrence, and (iii) processing with memoization.

3.3.1. Problem Discretization. The problem of SATO is solved in multiple stages by discretization, and each stage works for one segment. For each stage, the initial trajectory strategy (including speed and flight level of the cruise) which has not been adjusted when entering the corresponding segment is defined as its state. Given a stage with a specific state, there is a decision set from which one action should be selected and performed. The optional actions in the decision set can be classified into three types as shown in Table 1, and all of them should be accessed so that the optimal one can be identified according to the predefined objective function and constraints.

For the action types of both Type $(1,0)$ and Type $(0,1)$, there are a certain number of options to be selected from according to the difference in adjustments. The selected action will further determine the initial state at the next stage. Figure 1 illustrates the state transition process from stage $j$ to the next one $j+1$.

In this figure, $S_{j}$ is the state of the current stage $j$. The optional actions are distinguished by vectors with a structure like $\left\{v_{j}, h_{j}\right\}$ to indicate the decision about speed and flight level. Specifically, the action of $\left\{v_{j}^{0}, h_{j}^{0}\right\}$ means neither the speed nor flight level is changed compared with the initial strategy, namely, the action of Type $(0,0)$. Classified into different types, $\left\{v_{j}^{\prime}, h_{j}^{0}\right\}$ and $\left\{v_{j}^{0}, h_{j}^{\prime}\right\}$ represent adjusting the speed to $v_{j}^{\prime}$ and adjusting the flight level to $h_{j}^{\prime}$, respectively. It is worth noting that $v_{j}^{\prime}$ cannot take the value of $v_{j}^{0}$ and $h_{j}^{\prime}$ cannot take the value of $h_{j}^{0}$. There are $n$ possible states for stage $j+1$ in total with correspondence to the selected options, and the value of $n$ depends on the bounds defined by equations (25) and (26). $r_{1}, r_{2}, r_{3}$, and $r_{4}$ are all ordinal numbers valuing between 1 and $n$. The executed action onstage $j$ determines the initial state of stage $j+1$ as equations (32) and (33) describe.

$$
\begin{aligned}
& v_{j+1}^{0}=v_{j}, \\
& h_{j+1}^{0}=h_{j} .
\end{aligned}
$$

3.3.2. Forward Recurrence. In the discrete dynamic system, as Figure 1depicts, the effectiveness of the DP method depends on the recurrence relation between stages. The optimal substructure of a multistage decision-making problem makes the optimal policy to be usually obtained by a step-by-step backward process according to Bellman's principle of optimality [35]. If the optimal action sequence over the stage horizon of $j$ is expressed as $\pi_{j}^{*}\left(S_{j}\right)$, the backward process means that computing $\pi_{j}^{*}\left(S_{j}\right)$ requires $\pi_{j+1}^{*}\left(S_{j+1}\right)$, for which
TABle 1: Different types of action at each stage.

\begin{tabular}{lc}
\hline Action type & Description \\
\hline Type $(0,0)$ & Neither the speed nor flight level is adjusted. \\
Type $(1,0)$ & The speed is adjusted while the flight level is not. \\
Type $(0,1)$ & The flight level is adjusted while the speed is not.
\end{tabular}

$\pi_{j+2}^{*}\left(S_{j+2}\right)$ has to be calculated and so forth, until $j$ reaches the final stage $N_{j}$. As the stage number increases, the number of states within a stage will grow exponentially. For the state of the final stage is usually unknown when performing the backward process, all possibilities need to be considered and precalculated, and dealing with them in an exponential space will be of great challenge [36]. That prompts us to employ a forward induction approach to tackle the proposed SATO problem in a different way.

The optimal solution obtained by computing backward can be also acquired through forward recurrence, by which the intermediate states are only considered when needed rather than being all precalculated. In the forward induction manner, the optimal sequence between the initial stage and the current stage being tested is continuously computed, which is repeated until the optimal actions for all stages over the whole decision horizon are determined. In this paper, the forward induction is performed to realize SATO, and the adopted forward recurrence formula is presented in

$$
O_{j+1}^{*}\left(S_{j+1}\right)=\max \left\{O_{j}^{*}\left(S_{j}\right)+C_{j}\left(v_{j}, h_{j}\right)\right\},
$$

where $C_{j}\left(v_{j}, h_{j}\right)$ is defined as the conversion value, representing the performance contribution when transitioning from state $S_{j}$ to state $S_{j+1}$ by adopting the cruise strategy of $\left\{v_{j}, h_{j}\right\}$ on the segment $j . O_{j}^{*}\left(S_{j}\right)$ is the accumulated comprehensive performance of SATO using the optimal trajectory strategy over the stage horizon of $j$, namely, the objective function value of taking $\pi_{j}^{*}\left(S_{j}\right)$ as the action sequence. In order to calculate $O_{N_{j}}^{*}\left(S_{N_{j}}\right)$, which is the optimal accumulated performance over the whole decision horizon, all intermediate values are about $O_{j}^{*}\left(S_{j}\right)$ where $j$ values between 1 and $N_{j}-1$ are required. Finally, there comes the optimal action sequence over the whole decision horizon, namely, the preferred trajectory strategy on the whole cruise route as

$$
\pi_{N_{j}}^{*}\left(S_{N_{j}}\right)=\arg \max \left\{O_{N_{j}}^{*}\left(S_{N_{j}}\right)\right\} .
$$

3.3.3. Processing with Memoization. Although the forward recurrence approach has advantages in solving the problem with an unknown final state, there are still a lot of possible intermediate states between the initial and the final stage to deal with. Figure 1only exemplifies one case where there is an exact state, $S_{j}$, for stage $j$ and there are $n$ different possibilities for $S_{j+1}$. However, considering other conceivable states of stage $j$, there exist duplications in the possible values of $S_{j+1}$ since all possible actions are constrained within the same decision space, and different values of speed or flight level may become equal after different amounts of adjustment. It means that quite a large number of calculations are repeated during the forward induction process, 


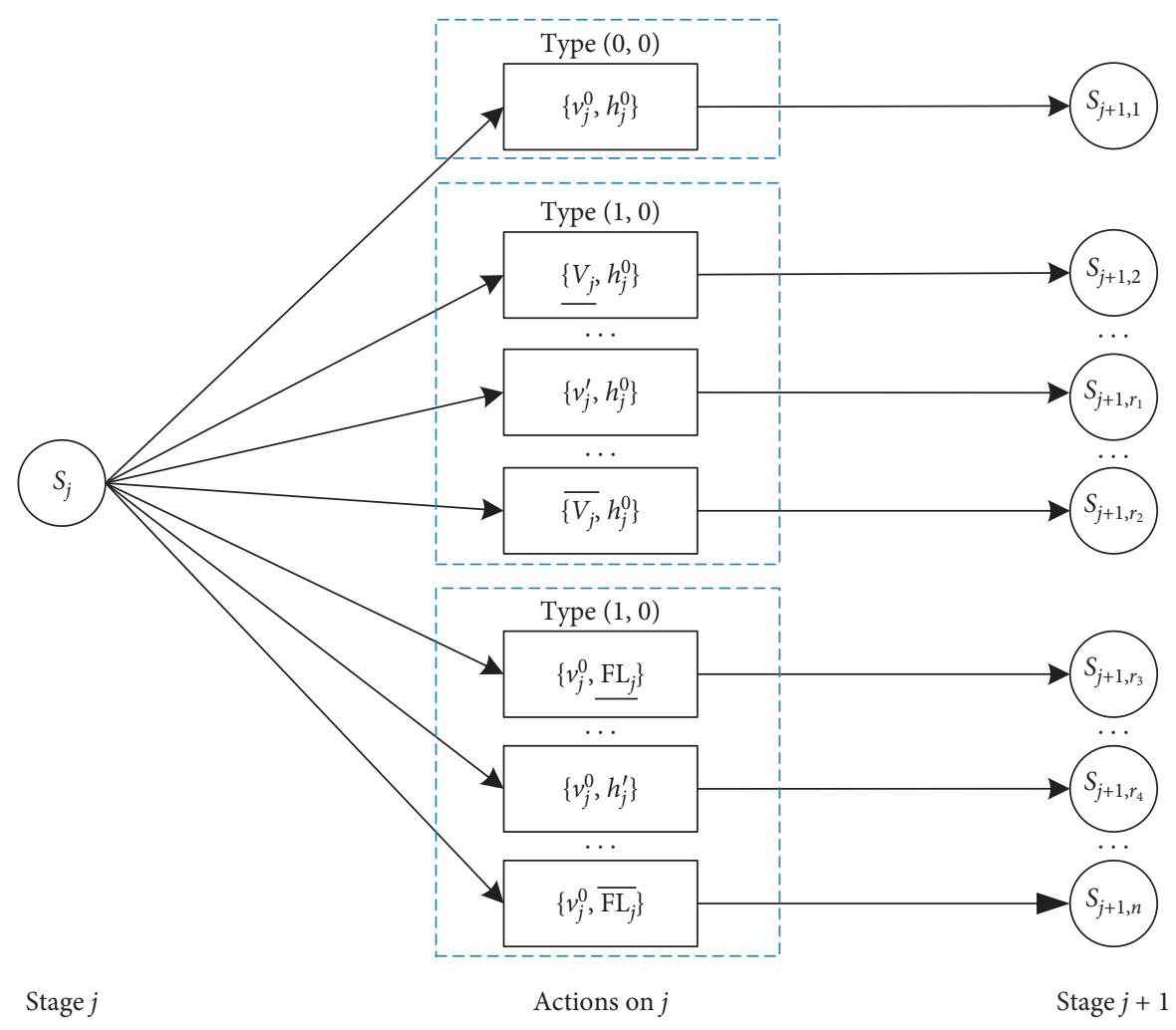

Figure 1: Process of state transition from one stage jto the next stage $j+1$.

for which we come up with the idea that introducing memoization technology to store the computed values and further improve the computational efficiency.

The improved approach called memoization dynamic programming (MDP) avoids duplicated calculation by the establishment and usage of two memos: one stores the intermediate values and the other stores the conversion values. In the course of forward processing based on equation (34), all needed intermediate values and conversion values will be first looked up according to defined keywords in the corresponding memos. If they have existed, the values will be directly extracted and used; otherwise, the computation is executed and the calculated results are stored into memos for later use.

\section{Case Study}

As the busiest domestic one in China according to the statistics of the Civil Aviation Administration of China (CAAC), the flight route from Beijing Capital International Airport (ICAO Code: ZBAA) to Shanghai Hongqiao International Airport (ICAO Code: ZSSS) is taken as an example. Real-world operational data about it are collected and processed to apply the proposed SATO scheme. All computations have been run on a computer with a 64-bit Intel i710700@2.90 GHz six-core CPU, RAM of 8 GB, and Windows 10 . The numerical results and performance analysis are given in this section.
4.1. Data and Materials. Figure 2 shows the key points on route ZBAA-ZSSS. The cruise phase studied in this paper includes 16 segments from ELKUR to SANSA and spans about 471 nautical miles. From December 1, 2020, to December 31, 2020, a total of 1265 flights were operated on route ZBAA-ZSSS using aircraft of 26 types. Those operated by Airbus 320-100 (A321), Airbus 330-300 (A333), Boeing 737-800 (B738), Boeing 777-300ER (B77W), and Airbus 330-200 (A332), which together account for about $81.19 \%$ of the total, are studied in this paper to calculate and analyze the performance of SATO. The data above about historical flights was provided by the East China Air Traffic Management Bureau CAAC.

As for the original cruise strategies of the studied flights working as contrast in the calculation, they were extracted from the ADS-B tracking data. In the optimization process, it is confined that the strategy in the first segment (ELKUR-DOXAB) should be in accordance with the original one to determine the initial state in the first stage, and the allocation principle of flight level in China is followed constantly. The real-time weather data during flight operation was obtained through spatial reverse weighting and horary linear interpolation based on the sounding meteorological information published by the University of Wyoming.

Additionally, the required performance parameters of different types of aircraft are shown in Table 2 according to BADA. The involved macroeconomic data used to calculate 


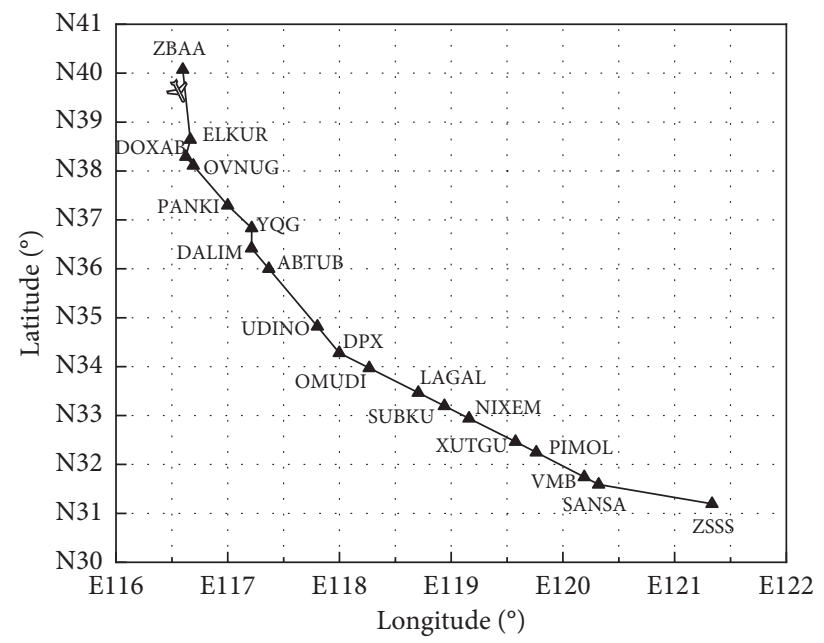

Figure 2: Location of the key points on route ZBAA-ZSSS.

TABLE 2: Aircraft performance parameters of different types of aircraft according to BADA.

\begin{tabular}{lccccc}
\hline Parameter & & Aircraft type & \\
& A321 & A333 & B738 & B77W & A332 \\
\hline$S\left(\mathrm{~m}^{2}\right)$ & 122.60 & 361.60 & 124.65 & 427.82 & 361.60 \\
$m(\mathrm{~kg})$ & 72000 & 174000 & 65300 & 290300 & 190000 \\
$C_{f c r}$ & 1.00000 & 0.93655 & 0.92958 & 0.92881 & 0.95422 \\
$C_{f_{1}}$ & 0.72987 & 0.61503 & 0.70057 & 0.54435 & 0.59426 \\
$C_{f_{1}}$ & 1236.90 & 919.03 & 1068.10 & 876.21 & 825.84 \\
$C_{D_{0}}, \mathrm{CR}$ & 0.026984 & 0.019805 & 0.025452 & 0.022913 & 0.018953 \\
$C_{D_{2}, \mathrm{CR}}$ & 0.035074 & 0.031875 & 0.035815 & 0.042347 & 0.032965 \\
\hline
\end{tabular}

VTT were collected from the 2020 National Economic and Social Development StatisticalBulletin of China. The passenger number of each flight was obtained by combining the seat number of aircraft and the average load factor on the route in historical operation.

4.2. Numerical Results and Discussion at Different Levels. Using the standard values for parameters as Table 3 shows, benchmark experiments have been conducted for one flight, one day, and one month, respectively, to explore the performance of the proposed SATO scheme at different levels.

4.2.1. For One Flight. The optimization was firstly carried out for the flight CHH7605 operated by A738 and departing at $07: 20$ on December 1, 2020. Note that all time described in this paper uses the standard of Beijing time $(\mathrm{UTC}+8)$. With the original cruise strategy of $409 \mathrm{kt}$ for speed and 33,100 ft for flight level, the proposed MDP algorithm worked effectively on CHH7605 in obtaining solutions and the time consumption was about $75 \mathrm{~s}$, which was significantly smaller than that before introducing memorization (about $342 \mathrm{~s}$ ).

Results show that the total social cost of flight $\mathrm{CHH7605}$ in the whole cruise route decreases from $\$ 8861.32$ to $\$ 8210.13$ through optimization. It means a CBI of $\$ 651.19$, and specifically, the values of EBI, GBI, and PBI are $\$ 137.62$, $\$ 59.26$, and $\$ 454.31$, respectively. The optimized cruise
TABle 3: Standard values of parameters used in benchmark experiments.

\begin{tabular}{lc}
\hline Parameter & Standard value \\
\hline$C_{F}(\$ / \mathrm{kg})$ & 0.87 \\
$\mathrm{SCC}(\$ / \mathrm{t})$ & 25 \\
$\mathrm{CI}(\mathrm{kg} / \mathrm{min})$ & 35 \\
$\mathrm{LF}(\%)$ & 85 \\
$p_{b}(\%)$ & 65 \\
$H($ years $)$ & 50 \\
$m_{v}(\mathrm{kt})$ & 9.72 \\
$\mu_{v}(\mathrm{kt})$ & 0.97 \\
$m_{h}(\mathrm{kt})$ & 2000 \\
$\mu_{h}(\mathrm{kt})$ & 2000 \\
\hline
\end{tabular}

strategy for $\mathrm{CHH} 7605$ is illustrated in Figure 3, in which different flight levels in 16 segments are separated by grid lines, and the shaded parts represent contrails formed in those areas during the corresponding period of flight operation.

Figure 4 shows the original and optimized social cost of CHH7605 on different segments and also illustrates the per nautical mile values of economic benefit $(\mathrm{EBN})$, green benefit $(\mathrm{GBN})$, and passenger benefit $(\mathrm{PBN})$. It can be observed that, in addition to the first segment with strategy unchanged, the social costs in all other segments dropped effectively after optimization. Furthermore, from the perspective of various benefits per mileage, positive 


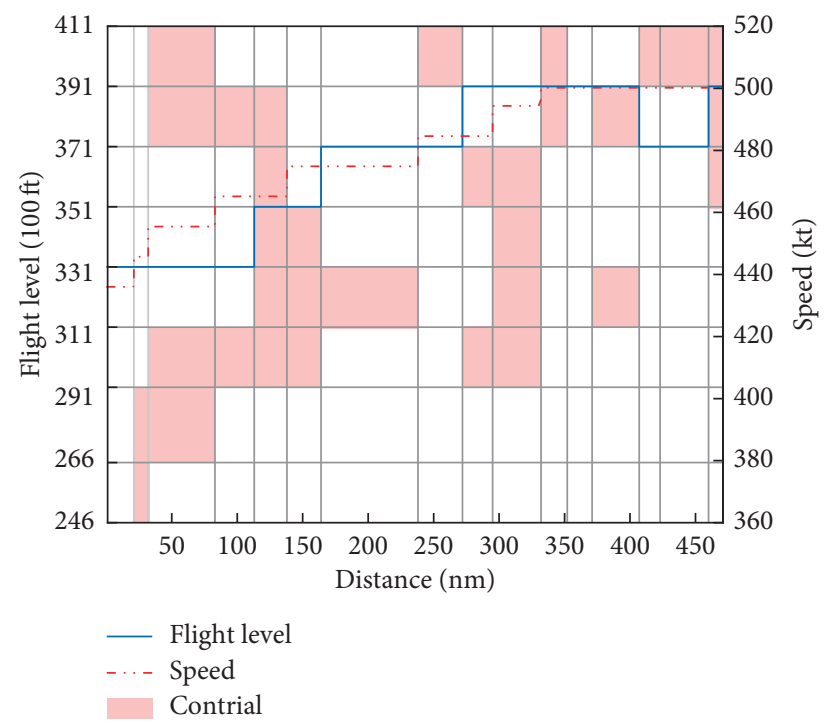

Figure 3: The optimized cruise strategy for flight CHH7605 on December 1, 2020.

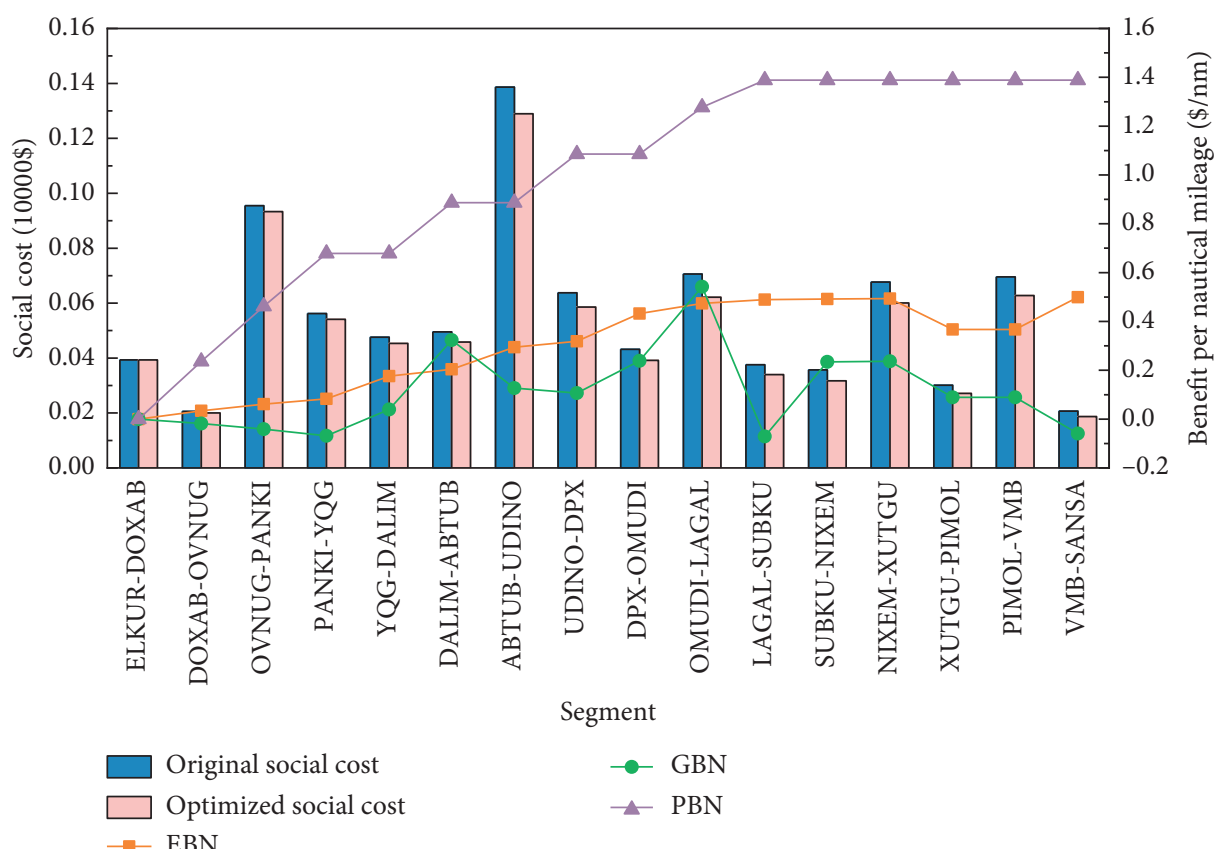

FIgURE 4: The original and optimized social cost of $\mathrm{CHH7605}$ and various benefits per nautical mileage of SATO in different segments.

performance is shown on each segment in achieving both economic benefit and passenger benefit, while environmental benefits are sacrificed sometimes. Combined with the strategy illustrated in Figure 3, the trends of curves in Figure 4 reflect that EBN is significantly affected by the selected flight level, and PBN has a strong positive correlation with the speed strategy.

4.2.2. For One Day. On December 1, 2020, there were 30 historical flights operated by the mentioned 5 types of aircraft and tracked by ADS-B on route ZBAA-ZSSS (37 in total). Experiments were conducted on these 30 flights with one day divided into consecutive periods that all span one hour. The flights that departed in the same period were optimized adopting one same set of meteorological data. Results show that at the one-day level, the optimization brings a fuel saving of about $3653.90 \mathrm{~kg}$ in total, and a comprehensive benefit value of about $\$ 32660.19$ can be achieved, including \$6644.59 for EBI, \$3772.11 for GBI, and $\$ 22243.51$ for PBI. Figure 5 presents the results of 30 flights in terms of their original social cost as well as the optimal comprehensive benefit (CBI) obtained from SATO. The maximum CBI and minimum CBI are marked in the figure in which a larger circle represents a greater CBI for the flight, and the aircraft types are distinguished by the usage of 


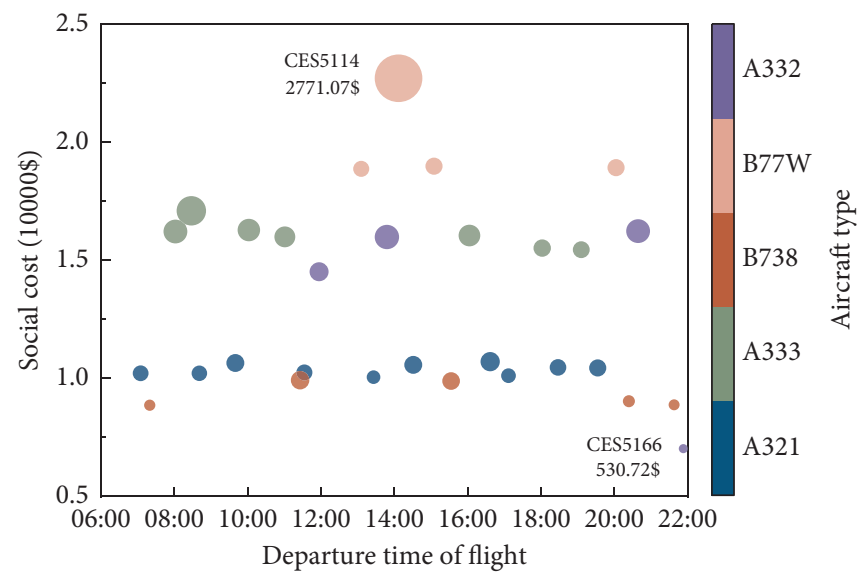

Figure 5: The social cost of 30 flights on December 1, 2020, and their results about comprehensive benefits from SATO.

colors. It can be observed that in most periods on December 1, 2020, the flights operated by B77W, A333, and A332 generated relatively higher social costs compared with A321 and B738. Given a certain type of aircraft, the social cost and optimal CBI will vary with the difference in operating duration because of the changes in meteorological conditions and the generated greenhouse effect.

The per capita performance of SATO was further calculated combined with the passenger number of each flight. From a holistic perspective of 30 flights, the per capita fuel saving (FSP) value is about $0.62 \mathrm{~kg}$, and the per capita comprehensive benefit (CBP) value is about $\$ 5.56$ and is composed of $\$ 1.13$ for per capita economic benefit (EBP), $\$ 0.64$ for per capita green benefit (GBP), and $\$ 3.79$ for per capita passenger benefit (PBP). Table 4 presents the results of different aircraft types on December 1, 2020. It shows that the scheme results in a greater $\mathrm{CBP}$ for $\mathrm{A} 321$ or B738 in spite of the relatively smaller social cost (as Figure 5 illustrates). However, it shows poor efficiency for A333 because all indicators about per capita performance value are lower than the average level in the holistic perspective. A332 is the one with the closest results to the average of 30 flights. Interestingly, under the premise that CBP does not deviate too far from the average value (\$5.56), the scheme brings a significantly smaller FSP and GBP and a relatively higher PBP for B738, while the opposite phenomenon is shown for B77W. That indicates the tradeoff between different performances in SATO and enables us to infer that the economic benefit of SATO comes from fuel savings for B77W, but from the operating time reduction which contributes to PBP for B738.

4.2.3. For One Month. The simulation was also applied to the entire month of December 2020 (1026 flights), with the standard parameters. Additionally, experiments with a time horizon of 25 and 100 years were also implemented. Figure 6 illustrates the flight number on each day and the daily comparison of social cost. Using the proposed SATO scheme, a reduction in social cost can be found on each day of December 2020, with different time horizons, accumulating to the monthly CBI of about $\$ 2,102,248, \$ 1,188,471$, and $\$ 1,100,686$ with the time horizon of 25,50 , and 100 years, respectively. At the same time, it is not surprising to find that the social cost on a certain day (such as December $22,2020)$ is relatively pronounced compared to those days (December 10, 2020, and December 15, 2020) with a similar or larger flight number because of worse weather conditions.

In terms of the composition of the monthly comprehensive benefit from SATO, statistics of EBI, GBI, and PBI classified by aircraft type with different time horizons are given in Figure 7, in which results about the comprehensive benefit rate (CBR), defined as the ratio of $\mathrm{CBI}$ to the original social cost, are illustrated to compare the relative efficiency of SATO in achieving comprehensive benefit. As indicated in Figure 7, the comprehensive performance of SATO derives mainly from the green benefit with the 25 -year time horizon and from the passenger benefit with the time horizons of 50 and 100 years. There exists a difference in CBR between various aircraft types. However, compared with the other two cases in Figures 7(a) and 7(c), the difference is not so significant when $H$ value is 50 in Figure 7(b) although A321 and B738 still show relatively larger values of CBR. It indicates that the scheme performs better in achieving comprehensive benefit for A321 and B738 with the 50-year time horizon, which is consistent with the daily results about CBP presented in Table 4.

\subsection{Robustness in Obtaining Comprehensive Benefit.} There is no reason to doubt that the numerical results above will vary with the change of calculation parameters. Some of the parameters are related to the specific operating background. For example, the cost index (CI), the load factor (LF), and the proportion of business passengers $\left(p_{b}\right)$ are distinguished by the operational preferences of airlines, the utilization efficiency of aircraft, and the characteristics of the route market, respectively. In order to test the robustness of the proposed SATO scheme, three sets of comparative tests were carried out with different values of CI, LF, and $p_{b}$, respectively, using the 1026 monthly flights with a 50-year time horizon. It is worth mentioning that, in each set of tests, all parameters other than the one to be studied are equal to the standard values presented in Table 3. 
TABLE 4: The statistics of flights and per capita performance of SATO for different aircraft types on December 1, 2020.

\begin{tabular}{|c|c|c|c|c|c|c|c|}
\hline \multirow{2}{*}{ Aircraft type } & \multirow{2}{*}{ Flight number } & \multirow{2}{*}{ Passenger number } & \multicolumn{5}{|c|}{ Per capita performance of SATO } \\
\hline & & & FSP $(\mathrm{kg})$ & $\operatorname{EBP}(\$)$ & GBP $(\$)$ & PBP (\$) & $\mathrm{CBP}(\$)$ \\
\hline A321 & 10 & 1530 & 0.46 & 1.26 & 0.52 & 4.48 & 6.25 \\
\hline A333 & 7 & 1761 & 0.49 & 0.85 & 0.48 & 3.69 & 5.03 \\
\hline B738 & 5 & 701 & 0.20 & 1.11 & 0.23 & 4.48 & 5.82 \\
\hline $\mathrm{B} 77 \mathrm{~W}$ & 4 & 1074 & 1.35 & 1.45 & 1.37 & 2.50 & 5.31 \\
\hline A332 & 4 & 806 & 0.63 & 1.10 & 0.62 & 3.80 & 5.52 \\
\hline
\end{tabular}

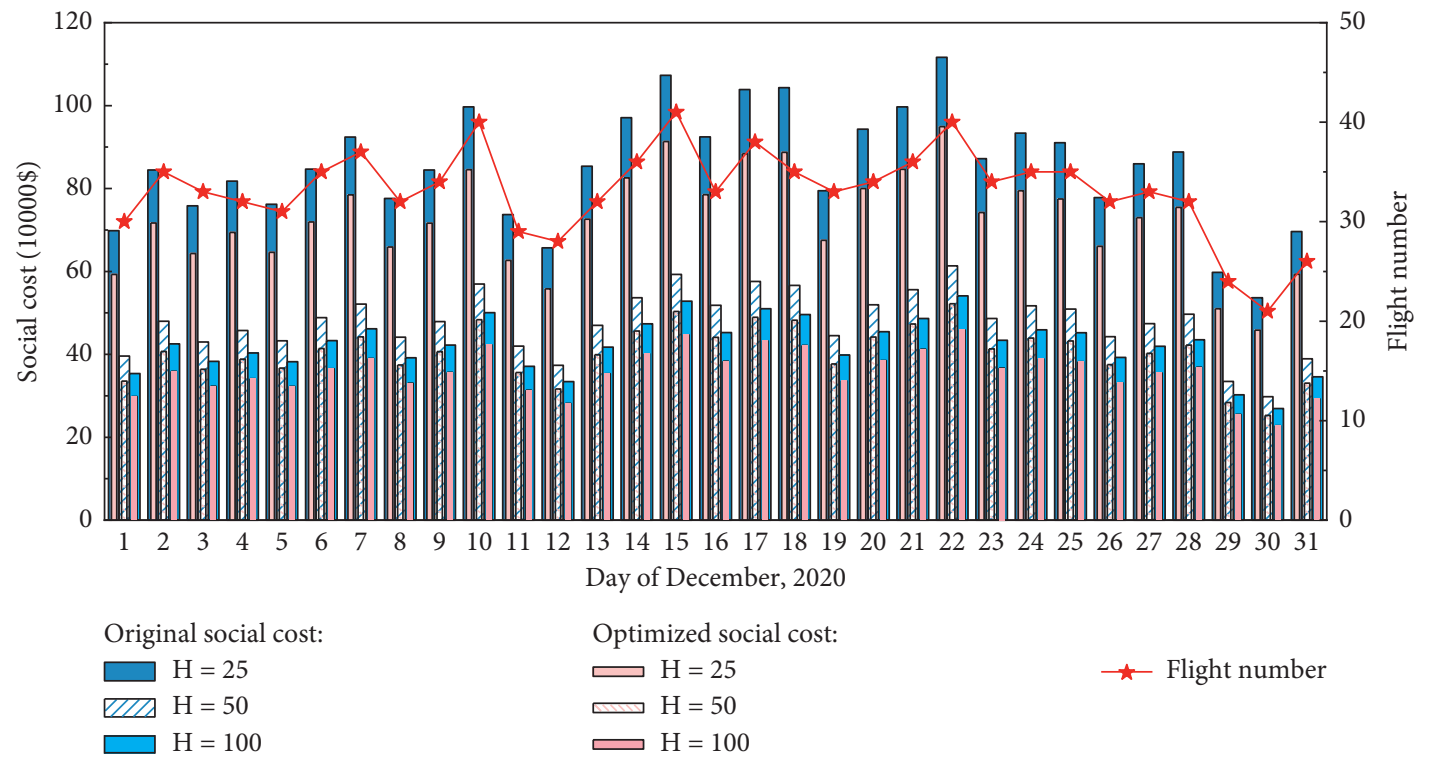

Figure 6: Flight number on route ZBAA-ZSSS and comparison of social cost with different time horizons on all days of December 2020.

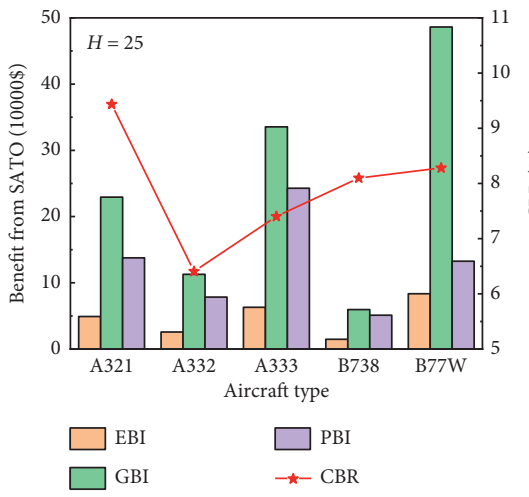

(a)

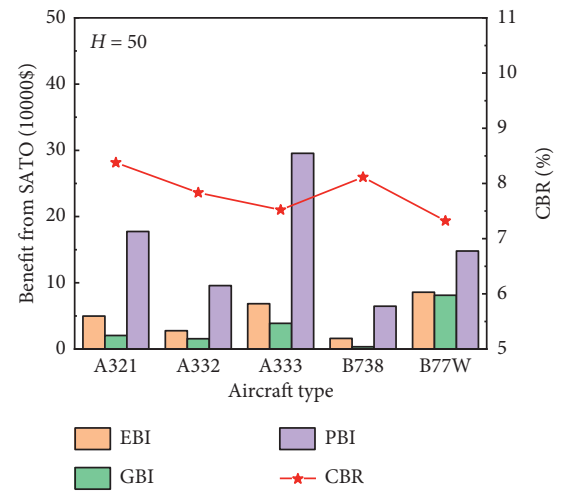

(b)

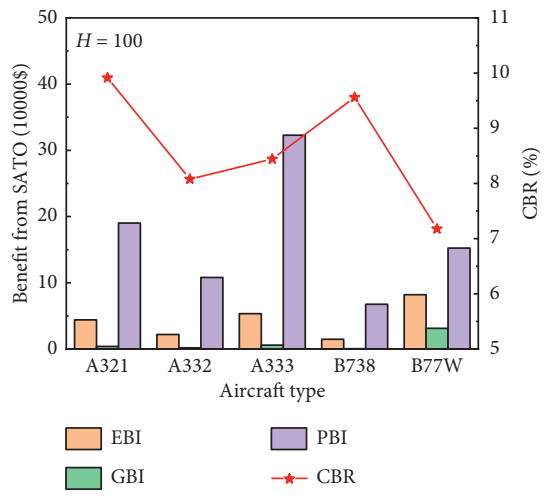

(c)

FIgure 7: Monthly EBI, GBI, PBI, and CBR classified by aircraft type with different time horizons.

Figure 8 shows the results of the three sets of tests. Similar to the comprehensive benefit rate (CBR), there are economic benefit rate (EBR), green benefit rate (GBR), and passenger benefit rate (PBR) defined and employed to indicate the relative efficiency of SATO in terms of different performance. In general, CBR does not fluctuate greatly with the changes of operating related parameters with the time horizon of 50 years, and all possible values of $p_{b}$, the parameter which shows the most significant variation in results, still guarantee CBR to value within a small range of $7 \%-8.5 \%$. That indicates that the proposed SATO scheme will perform robustly in obtaining comprehensive benefits (reducing social cost) under diverse operating backgrounds. Another interesting finding is that CBR is relatively higher for the business route (with a lager $p_{b}$ ) when the load factor is about $85 \%$. Furthermore, the trend of CBR is most 


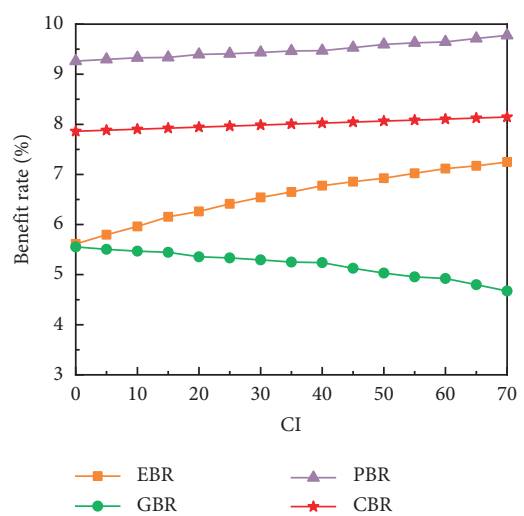

(a)

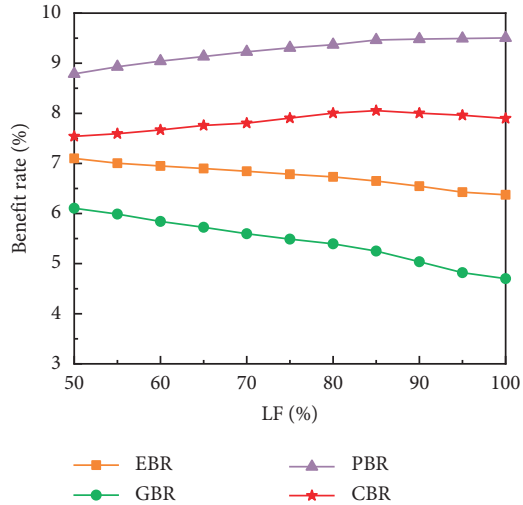

(b)

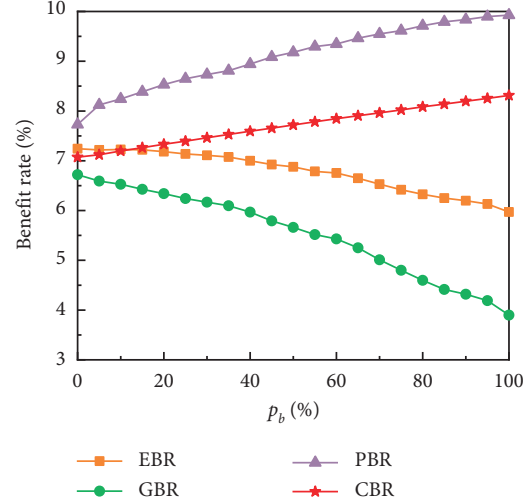

(c)

FIgURE 8: Comparative monthly results about SATO performance with different values of CI, LF, and $p_{b}$.

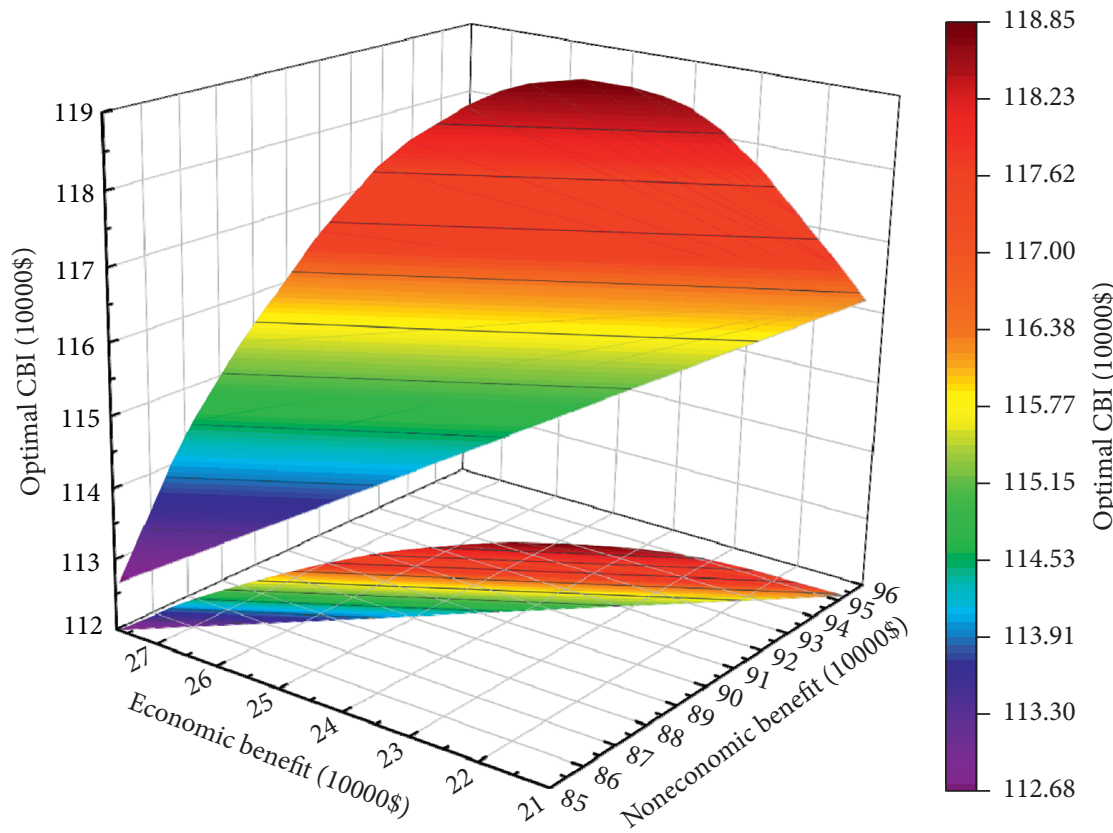

Figure 9: Tradeoff between the economic and noneconomic benefit in achieving maximum comprehensive benefit (at the monthly level using standard values for parameters).

consistent with that of PBR, and there are always tradeoffs between EBR and GBR or PBR in all studied cases. It means the benefit to passengers which has never introduced into ATO work is an important component of the social benefit, and various subjects should all attract attention to enhance the integrated sustainability of air transport.

\subsection{Tradeoffbetween the Economic and Noneconomic Benefits.} The previous results and analysis have shown the effectiveness and robustness of the proposed SATO scheme in achieving comprehensive benefits. However, it is difficult to ensure that decision makers consider the noneconomic benefit which does not directly contribute to airlines' operating profits like economic benefit and plays an essential role in the social development in practical applications. By adjusting the weight of EBI in optimization, we explore the tradeoff relationship between economic savings and noneconomic benefit in achieving maximum comprehensive benefit at the monthly level with all parameters set as standard values in this paper. Figure 9 shows the results of the noneconomic benefit (the sum of GBI and PBI) and the optimal comprehensive benefit (CBI) when the economic performance (EBI) gradually decreases from its maximum possible value (about $\$ 276,500$ ), which is obtained with pure economic concern.

As shown in Figure 9, the sacrifices of economic benefit can bring a gradual slowing increase in noneconomic benefit until EBI decreases to approximately $\$ 225,000$, making the obtained noneconomic benefit increase from $\$ 850,000$ to $\$ 952,000$, and after that, additional economic costs will hardly contribute to more noneconomic benefit. The 
maximum (about $\$ 1,188,500$ ) of the optimal CBI is obtained when the EBI value is around $\$ 250,000$, which means an addition of about $\$ 26,500$ in economic costs compared with the results (about $\$ 276,500$ ) under economic priority. The findings of the tradeoff can provide support for the decision makers and practitioners who committed to sustainable air transport.

\section{Conclusions}

The problem of sustainable aircraft trajectory optimization for the cruise phase is modeled based on an innovative performance framework and solved using memoization dynamic programming. The established SATO performance framework focuses on the comprehensive social benefit by taking both economic benefit and noneconomic one into consideration, so as to enhance the integrated sustainability including economic, environmental, and marketing aspects. Combining a forward induction approach with the usage of two memos, a method of memoization dynamic programming is designed to obtain a reliable solution with improved efficiency. Through the application to the flight route of ZBAA-ZSSS, there are some conclusions about the SATO scheme as follows:

(1) Considerable comprehensive social benefit can be obtained through the SATO scheme at the level of one flight, one day, or one month, and the scheme also shows competency with different time horizons in the monthly application

(2) Both the aircraft type and the meteorological condition can affect the comprehensive benefit efficiency of SATO, and the difference resulting from aircraft type with the 50 -year time horizon is not so significant as that of 25 or 100 years

(3) The scheme shows robustness in comprehensive performance under different operational backgrounds with a stable CBR always staying at around $8 \%$, and it is relatively better when applied to the business route with a load factor of about $85 \%$

(4) With the abandonment of economic benefit during optimization, the noneconomic benefit is found to grow at a slowing rate which approaches 0 eventually, and the optimal comprehensive performance (about $\$ 1,188,500$ ) can be obtained when accepting a $\$ 26,500$ increase in economic costs

This study contributes to sustainable air transport by the improved model and solution method for aircraft cruise trajectory optimization. Future research can explore an overall scheme of SATO considering all flight phases including climb, cruise, and descent. The sensitivity analysis and tradeoff exploration with more time horizons also produce research opportunities.

\section{Data Availability}

The data used to support the findings of this study are available from the first author upon request.

\section{Conflicts of Interest}

The authors declare that there are no conflicts of interest.

\section{Acknowledgments}

This work was funded by the National Natural Science Foundation of China (grant number U1933119), the Foundation of State Key Laboratory of Air Traffic Management System and Technology (grant number SKLATM202003), and the Foundation of the Graduate Innovation Center, Nanjing University of Aeronautics and Astronautics (grant number kfjj20200735). Grateful thanks are also extended to East China Air Traffic Management Bureau CAAC for the support in data collection.

\section{References}

[1] R. Chai, A. Savvaris, A. Tsourdos, Y. Xia, and S. Chai, "Solving multiobjective constrained trajectory optimization problem by an extended evolutionary algorithm," IEEE Transactions on Cybernetics, vol. 50, no. 4, pp. 1630-1643, 2020.

[2] N. N. Zhao, N. Li, Y. Sun, and Z. Gao, "4D trajectory planning of aircraft taxiing considering time and fuel," Discrete Dynamics in Nature and Society, vol. 2020, Article ID 9603968, 12 pages, 2020.

[3] A. Cuspilici, P. Monforte, and M. A. Ragusa, "Study of Saharan dust influence on $\mathrm{PM}_{10}$ measures in Sicily from 2013 to 2015," Ecological Indicators, vol. 76, pp. 297-303, 2017.

[4] G. P. Brasseur and M. Gupta, "Impact of aviation on climate: research priorities," Bulletin of the American Meteorological Society, vol. 91, no. 4, pp. 461-464, 2010.

[5] S. Marquart, M. Ponater, F. Mager, and R. Sausen, "Future development of contrail cover, optical depth, and radiative forcing: impacts of increasing air traffic and climate change," Journal of Climate, vol. 16, no. 9, pp. 2890-2904, 2003.

[6] S. Hartjes, T. Hendriks, and D. Visser, "Contrail mitigation through 3D aircraft trajectory optimization," in Proceedings of the 16th AIAA Aviation Technology, Integration, and Operations Conference, Washington, DC, USA, 2016.

[7] Y. Tian, L. L. Wan, B. J. Ye, and D. W. Xing, "Cruise flight performance optimization for minimizing green direct operating cost," Sustainability, vol. 11, no. 14, p. 3899, 2019.

[8] Y. Tian, X. He, Y. Xu, L. Wan, and B. Ye, "4D trajectory optimization of commercial flight for green civil aviation," IEEE Access, vol. 8, pp. 62815-62829, 2020.

[9] W. Liu, X. Liang, Y. Ma, and W. Liu, "Aircraft trajectory optimization for collision avoidance using stochastic optimal control," Asian Journal of Control, vol. 21, no. 5, pp. 2308-2320, 2018.

[10] Y. Saito, S. Yoshimura, M. Inoue, M. Takahashi, and Y. Sugihara, "Optimal trajectory planning of aircraft for fairsharing of noise," IFAC-PapersOnLine, vol. 52, no. 8, pp. 271-276, 2019.

[11] M. Kos Koklic, M. Kukar-Kinney, and S. Vegelj, “An investigation of customer satisfaction with low-cost and fullservice airline companies," Journal of Business Research, vol. 80, pp. 188-196, 2017.

[12] M. Kelly, "An introduction to trajectory optimization: how to do your own direct collocation," SIAM Review, vol. 59, no. 4, pp. 849-904, 2017.

[13] D. S. Zachary, J. Gervais, and U. Leopold, "Multi-impact optimization to reduce aviation noise and emissions," 
Transportation Research Part D: Transport and Environment, vol. 15, no. 2, pp. 82-93, 2010.

[14] S. Khardi, "Applied methods validating aircraft flight path optimization. Theoretical and experimental considerations," Applied Mathematical Sciences, vol. 7, no. 45, pp. 2209-2228, 2013.

[15] Y. X. Lim, A. Gardi, and R. Sabatini, "Optimal aircraft trajectories to minimize the radiative impact of contrails and $\mathrm{CO}_{2}$," in Proceedings of the 1st International Conference on Energy and Power, Melbourne, Australia, 2017.

[16] S. Hartjes and H. Visser, "Efficient trajectory parameterization for environmental optimization of departure flight paths using a genetic algorithm," Proceedings of the Institution of Mechanical Engineers, Part G: Journal of Aerospace Engineering, vol. 231, no. 6, pp. 1115-1123, 2016.

[17] V. Courchelle, M. Soler, D. González-Arribas, and D. Delahaye, "A simulated annealing approach to 3D strategic aircraft deconfliction based on en-route speed changes under wind and temperature uncertainties," Transportation Research Part C: Emerging Technologies, vol. 103, pp. 194-210, 2019.

[18] S. B. Zhu, G. X. Li, and J. W. Han, "Aircraft trajectory optimization in vertical flight profile based on improved particle swarm optimization algorithm," Journal of Jiangnan University (Natural Science), vol. 11, no. 2, pp. 163-168, 2012.

[19] Q. Mcenteggart and J. F. Whidborne, "Multiobjective environmental departure procedure optimization," Journal of Aircraft, vol. 55, no. 3, pp. 905-917, 2018.

[20] A. W. A. Hammad, D. Rey, A. Bu-Qammaz, H. Grzybowska, and A. Akbarnezhad, "Mathematical optimization in enhancing the sustainability of aircraft trajectory: a review," International Journal of Sustainable Transportation, vol. 14, no. 6, pp. 413-436, 2020.

[21] Z. F. Y. Wang, Y. Tian, L. L. Wan, and S. J. Ying, "Progress in the study of environmental impacts of high altitude flight," Environmental Protection Science, vol. 53, no. 3, pp. 100-105, 2017.

[22] Eurocontrol Experimental Center, Mode Accuracy Summary Report for the Base of Aircraft Data (BADA), Eurocontrol Experimental Center, Bretigny, France, 2012.

[23] Y. Tian, L. N. Ma, S. T. Yang, and Q. Wang, "A methodology for calculating greenhouse effect of aircraft cruise using genetic algorithm-optimized wavelet neural network," Complexity, vol. 2020, Article ID 7141320, 13 pages, 2020.

[24] B. Sridhar, N. Y. Chen, and H. K. Ng, "Fuel efficient strategies for reducing contrail formations in United States airspace," in Proceedings of the 29th Digital Avionics Systems Conference, Salt Lake City, UT, USA, 2010.

[25] J. S. Fuglestvedt, K. P. Shine, T. Berntsen et al., "Transport impacts on atmosphere and climate: metrics," Atmospheric Environment, vol. 44, no. 37, pp. 4648-4677, 2010.

[26] N. Y. Chen, B. Sridhar, H. K. Ng, and J. H. Li, "Evaluating tradeoff between environmental impact and operational costs for enroute air traffic," in Proceedings of the 2014 AIAA Guidance, Navigation, and Control Conference, National Harbor, MD, USA, 2014.

[27] S. Y. Jiang, X. L. Luo, and L. He, "Research on method of trajectory prediction in aircraft flight based on aircraft performance and historical track data," Mathematical Problems in Engineering, vol. 2021, Article ID 6688213, 11 pages, 2021.

[28] C. Q. Shao and Y. L. Chen, "Review of study on valuation of travel time," Journal of Beijing University of Technology, vol. 44, no. 3, pp. 417-423, 2018.
[29] F. G. F. da Silva and S. A. DeSouza, "Estimating travel time value by different data source using the logit model," Journal of Transport Literature, vol. 7, no. 4, pp. 107-129, 2013.

[30] L. Ding and X. Yang, "The response of urban travel mode choice to parking fees considering travel time variability," Discrete Dynamics in Nature and Society, vol. 2020, Article ID 8969202, 9 pages, 2020.

[31] A. M. H. Mahmudah, D. Sarwono, R. I. Pramesty, and P. S. Rahina, "Value of travel time for public transport passenger in urban and intercity trip," Applied Mechanics and Materials, vol. 845, pp. 408-415, 2016.

[32] G. S. Becker, "A theory of the allocation of time," The Economic Journal, vol. 75, no. 299, pp. 493-517, 1965.

[33] O. Banias, "Test case selection-prioritization approach based on memoization dynamic programming algorithm," Information and Software Technology, vol. 115, pp. 119-130, 2019.

[34] W. Bi, L. Tian, H. Liu, and X. Chen, "A stochastic dynamic programming approach based on bounded rationality and application to dynamic portfolio choice," Discrete Dynamics in Nature and Society, vol. 2014, Article ID 840725, 11 pages, 2014.

[35] M. Sedighizadeh, A. Mohammadpour, and S. M. M. Alavi, “A daytime optimal stochastic energy management for EV commercial parking lots by using approximate dynamic programming and hybrid big bang big crunch algorithm," Sustainable Cities and Society, vol. 45, pp. 486-498, 2019.

[36] Q. Deng, B. F. Santos, and R. Curran, "A practical dynamic programming based methodology for aircraft maintenance check scheduling optimization," European Journal of Operational Research, vol. 281, no. 2, pp. 256-273, 2020. 